\title{
Brumana: una maestra argentina que escribió para sus alumnos a partir de 1900*
}

\section{Resumen}

El presente artículo estudia a una mujer argentina y su labor docente e intelectual en un período crucial para la historia mundial. Este ejemplo dará lugar a analizar escuetamente la situación de la mujer en las postrimerías del siglo XIX junto a los cambios impulsados por la Rusia revolucionaria, acompañados estos por la aparición de luchas emprendidas por dichas féminas. Pretendemos entonces comprender algunas de las discusiones que resonaban a nivel mundial y centrarnos en una mujer en particular, quien con sus accionares profirió de manera escrita muchas de sus expresiones, poco permitidas en aquel entonces. Será Herminia Brumana la figura estudiada. Utilizando una de sus primeras obras, destinada para los alumnos junto a epístolas referentes, intentaremos encuadrar el pensamiento de una maestra que traspasó su labor educativa no solo con el objetivo de reflexionar sobre la misma, sino también para luchar por los derechos de los menos favorecidos.

\section{Palabras clave:}

Tesauro: mujer, maestra, educación, intelectual.

Autor: Argentina.

Referencia bibliográfica para citar este artículo: Mosso, Agustina Guadalupe. "Brumana: una maestra argentina que escribió para sus alumnos iniciándose el 1900". Anuario de Historia Regional y de las Fronteras, 24.2 (2019): 101-124.

Agustina Guadalupe Mosso: profesora y licenciada en Ciencias de la Educación de la Universidad Nacional del Rosario, Argentina. Becada por CONICET (Consejo Nacional de Investigaciones Científicas y Técnicas) ) al doctorado en Estudios de Género en la Facultad de Filosofía y Letras, de la Universidad de Buenos Aires. Código ORCID: 0000-0003-3515-7641. Correo electrónico: mossoagustina@gmail.com.

* Resultado de investigación en el marco del Doctorado Estudios de Género de la Facultad de Filosofía y Letras de la Universidad de Buenos Aires. 


\title{
Brumana: an Argentine Teacher who Wrote for her Students Beginning in 1900
}

\begin{abstract}
This article studies an Argentinean woman and her intellectual and teaching work at a crucial period for world history. This example will lead to a brief analysis of the situation of women in the late nineteenth century. Also, the changes promoted by revolutionary Russia, which were accompanied by the emergence of struggles undertaken by women. We intend to understand some of the discussions that resonated worldwide and study a woman. She uttered many of her expressions, not allowed at that time. Herminia Brumana will be the studied figure. Using one of his first works, we will try to frame the thought of a teacher who transferred her educational work with the aim of reflecting on it and fighting for the rights of the less favored.
\end{abstract}

Keywords:

Thesaurus: Woman, Teacher, Education, Intellectual. Author: Argentina.

\section{Brumana: uma professora argentina que escreveu para seus alunos a partir de 1900}

\section{Resumo}

Este artigo estuda uma mulher argentina e seu trabalho como professora e intelectual em um periodo crucial para a história do mundo. Este exemplo levará a uma breve análise da situação das mulheres no final do século XIX. Também as mudanças promovidas pela Rússia revolucionária, que foram acompanhadas pelo surgimento de lutas empreendidas por mulheres. Pretendemos entender algumas das discussões que ressoaram em todo o mundo e estudar uma mulher em particular, quem proferiu muitas de suas expressões, pouco permitidas naquele momento. Herminia Brumana será a figura estudada. Usando um de seus primeiros trabalhos, tentaremos enquadrar o pensamento de uma professora que transferiu seu trabalho educacional com o objetivo de refletir sobre ela e lutar pelos direitos dos menos favorecidos.

Palavras-chave:

Tesauro: mulher, professora, educação, intelectual. Autor: Argentina. 


\section{Párrafos introductorios}

Fue en el mes de febrero del año 1917 cuando una manifestación masiva de mujeres reunidas por el Día Internacional de la Mujer decide oponerse a la sangrienta guerra imperialista que llevaba ya tres años; más precisamente el día 23 del nombrado mes, obreras textiles de la ciudad de Petrogrado (San Petersburgo) decidieron unirse a la lucha que venían dando trabajadores y campesinos contra la guerra. Estas huelgas marcaron el inicio de la Revolución Rusa para muchos, dado que ellas provocaron la caída del zarismo, la formación de un gobierno provisional de corte liberal y el levantamiento de los soviets de obreros y campesinos que más tarde obtendrán todo el poder para exigir por paz, pan y tierra y así llevar adelante durante el décimo mes de ese año la Revolución. Es importante entonces resaltar aquí la labor de las trabajadoras, militantes bolcheviques, que salieron a la calle en busca del reconocimiento de derechos (al voto, al aborto libre y gratuito, al divorcio, a la despenalización de la prostitución y de la homosexualidad), mejores condiciones de vida y el surgimiento de profundos debates que van a producirse durante muchos años en el seno de la clase trabajadora.

Teniendo en cuenta el arriba descrito panorama internacional, y centrándonos en el tema educación, el que aquí nos convoca, resulta interesante poner en los inicios del siglo XX y ya situados en Argentina el desarrollo de diversas alternativas educativas vinculadas al posicionamiento político de sus protagonistas. Todas ellas se encuentran sostenidas por maestros socialistas (con sus propuestas de democratización del funcionamiento institucional) y anarquistas (formación en el trabajo, en principios científicos, anticlericales), quienes se encontraban influenciados por experiencias europeas, como el caso de la labor del conocido pedagogo Makarenko. Estuvieron sus supuestos pedagógicos vinculados a los ideales de los partidos comunistas, fundamentalmente a aquellos en defensa de la igualdad para la transformación de la realidad social. ${ }^{1}$ Igualmente, y en la misma localización geográfica, profundas críticas en el plano de la vinculación educación y sociedad vienen de la mano de Marx y Engels y se distribuyen hacia socialistas utópicos (como Owen y Fourier), que apuntan a una relación más estrecha entre educación y trabajo. Veremos a continuación a través del caso específico de una maestra del período: Herminia Brumana, cómo resuenan estas ideas en su oficio docente, pero también en el de escritura sobre las prácticas educativas.

En adelante, el lector se encontrará con algunos datos biográficos de la maestra antes mencionada. Luego, tendrá lugar el análisis de uno de sus libros en particular (observación: está atravesada por otros escritos referentes al libro como es el caso de las cartas que le enviaban a Brumana y ella respondía acerca de las opiniones que el libro emanaba, fragmentos de dicho ejemplar que la autora seleccionaba para publicar en revistas de gremios docentes). Por último, se darán a conocer unas reflexiones finales sobre el presente ensayo, focalizando la lucha emprendida por la maestra que elegimos estudiar con sus escritos como herramienta crucial.

${ }^{1}$ Adriana Puiggrós; Roberto Marengo, Pedagogías: reflexiones y debates. (Bernal, 2013) 53. 
Otro tema relevante que el artículo menciona en las próximas páginas refiere a la maestra como crítica de la realidad que aborda, pudiendo ser ubicada dentro de los movimientos de mujeres que hoy luchan por sus derechos, considerando injustas las relaciones de subordinación, demostrando una ferviente voluntad de cambiarlas. Convirtiéndose en "[...] figuras que según los accionares que desempeñaron pretendieron emancipar, liberar a las mujeres de la opresión”. ${ }^{2}$

Veremos cómo Brumana publicará escritos en los que se muestra comprometida socialmente con una lectura aguda e instantánea del acontecer político de su época. Analizaremos su primer libro para niños en edad escolar: Palabritas, y allí observaremos el modo en que dicha pedagoga prescribirá sentidos que pretenderá sean leídos y aprendidos en la cadencia del día a día escolar. Asimismo, advertiremos los sucesos que denotan la no aprobación formal de su obra para ser utilizada en las escuelas. Siendo sometido a concurso público, tal como lo disponía el artículo 57, inciso 15 de la Ley argentina de Educación No 1420 de 1884, Palabritas no obtendrá la aprobación; suceso que ignorará la autora y pasará a publicarlo dando a conocer entre colegas y amigos el contenido de su libro. A continuación, el lector podrá distinguir las lecturas que al interior del escrito Brumana decide exponer, muchas de las cuales discutirán la aparente igualdad social reinante en las escuelas. De este modo, lograremos comprobar cómo Herminia Brumana se convirtió en una educacionista que no se conformó con ejercer el magisterio, sino que además se animó a producir teorías, reflexiones, propuestas prescriptivas acerca de las prácticas educativas, construyendo una identidad laboral e intelectual propia, dentro del colectivo docente de su época.

\section{Una maestra llamada Herminia Brumana}

Nace un doce de septiembre de 1897 en Pigüé (pueblo ubicado a unos 500 kilómetros de Capital Federal) Herminia Brumana. Delia Piatti y Juan Brumana fueron sus progenitores, él prevenía del norte de Italia, ella, en cambio, era hija de italianos asentados en Tapalqué, otro pueblo de la campaña bonaerense. Justamente, el encuentro entre los padres de Herminia sucedió al calor del proceso migratorio propio de la Argentina de la bisagra de los siglos XIX y XX. Elegimos a su hija por considerarla una de las pocas maestras del período que se animó a cuestionar, en un momento caracterizado por transformaciones económicas, sociales, culturales, las ideas que giraban en torno a la feminidad, la maternidad, los modos de educar, los problemas de desigualdad social.

Esta docente debutará en el ámbito público gracias a dicha actividad laboral, a la vez que irá incursionando en la escritura (revistas, libros, ensayos, obras teatrales), destacando su vocación de educadora con elevado sentido de misión social, acreditada con espíritu renovador y progresista. Con lápiz y papel en mano, Brumana produjo escritos que no solo cuestionaron los ideales establecidos para aquel entonces sobre la condición femenina, sino además invitaron a reflexionar sobre el papel de las mujeres

\footnotetext{
${ }^{2}$ Marcela Nari. En Juan Suriano (comp.) La cuestión social en Argentina, 1870-1943. (Buenos Aires. La colmena. 2000) 277.
} 
en la época (como madres, maestras, trabajadoras) y sobre las escuelas, los niños. "En el período estudiado, las feministas desfilaron por la cornisa de la biología. Intentaron reformular la maternidad como una función social y política, pero sin abandonar la idea de la "naturaleza maternal" femenina". ${ }^{3}$ Podemos pensar a Brumana como una de ellas.

Herminia Brumana se recibe de maestra en la Escuela Normal Nacional de Olavarría, Buenos Aires, en el año 1916. Como decíamos antes, al mismo tiempo que ejercía la docencia emprendía su trabajo intelectual. Funda la revista literaria en su pueblo natal Pigüé y comienza a relacionarse con escritores y políticos porteños, quienes colaboran asiduamente con la publicación (revista social, de ideas y crítica). Entre los contribuyentes ubicamos a su futuro esposo Juan Antonio Solari, por entonces estudiante universitario comprometido con el socialismo y el movimiento estudiantil. "Ella también participó en las publicaciones de la agrupación anarcocomunista Insurrexit (1919-1920), de tendencia marxista-leninista, partidaria de la Revolución Rusa, pero con despliegues de tipo libertario". ${ }^{4}$ Dicha revista pugnaba por una reforma universitaria que incluyera al mundo obrero y de los trabajadores en los claustros. Como decíamos, Solari era parte del mismo grupo y, en el año 1921 se casa con Brumana convirtiéndose en padres de su único hijo al año siguiente. Las propuestas del Partido Socialista respecto a la protección del trabajo de las mujeres y los niños fueron claras. "Los socialistas consideraban que todos los ciudadanos nacen libres e iguales. Ellos formulaban una noción de ciudadanía universal que no excluía a las mujeres." 5

Desde el año 1917 hasta 1930 Brumana se radica en Buenos Aires y trabaja como maestra de grado. Luego, abandona los salones de clase para comenzar a ocupar diversos cargos en las escuelas hasta 1954, año en que fallece. Herminia Brumana fue una de las primeras escritoras argentinas que llevó a sus páginas una expectación social, dedicó la mayor parte de su delicado poder de observación e interpretación a la mujer argentina, enunciado con el que acuerdan muchas historiadoras feministas argentinas.

Finalizado el siglo XIX emergen nuevas ocupaciones femeninas a causa de los cambios ocurridos en el sistema económico mundial y entre ellas la docencia fue una singular labor asalariada que posibilitó a las mujeres a disponerse en el mercado. "Bien sabemos que en dicho periodo el trabajo femenino extra doméstico era observado con mucha discrepancia, incluso por los reformadores sociales que estaban emergiendo [...]" $]^{\prime 6}$ Podemos afirmar entonces que a Brumana el ingreso al magisterio le permitió desempeñarse a lo largo de toda su vida como escritora de artículos, libros, cuentos, cartas, notas de opinión. Y fue por este medio (principalmente el de

\footnotetext{
${ }^{3}$ Nari 287.

${ }^{4}$ Fletcher Lea, Una mujer llamada Herminia, (Buenos Aires. Catálogos Editora. 1987) 15.

${ }^{5}$ Mirta Lobato, Historia de las trabajadoras en la Argentina (1869-1960), (Bs. As. Edhasa, 2007) 211.

${ }^{6}$ Dora Barrancos, "Mujeres en la Argentina: un balance frente al bicentenario", (Revista de Trabajo. Año

6. Número 8. Buenos Aires, 2010) 325.
} 
la escritura militante) que conoció a quien fuera el padre de su hijo y esposo: Juan Antonio Solari, colaborador de la revista de temas literarios y sociales creada por Herminia (en Pigüé y que llevaba dicho nombre), a la vez que personalidad notable del socialismo vigente, como referíamos arriba. Desde la lógica del socialismo "el torbellino del capitalismo" había arrancado a las mujeres de sus "cárceles domésticas, poniéndolas en contacto directo con la lucha económica; “[...] cuando en el campo de esa lucha demostraron la misma eficiencia, el mismo rendimiento que el hombre $\{\ldots\}$ ya nadie discutió el derecho de la mujer de recobrar su personalidad y libertad".?

En el mes de agosto de 1918, a poco tiempo de obtener el título en una Escuela Normal de una localidad cercana a la suya, la maestra publicó su primer libro de lectura y que analizaremos más adelante: Palabritas. Tomaremos a esta mujer para representar con ella a otras que del mismo modo se atrevieron a participar en la primera mitad del siglo XX en discusiones donde las féminas quedaban por fuera. Brumana, ejerciendo el magisterio, logró dejar huellas en los archivos, ya que en calidad de alfabetizadora fue productora de obras donde la palabra escrita hoy permite historiarla. Brumana, a diferencia de otras maestras del período, produjo un vasto corpus documental que hoy se encuentra centralizado en el Centro de Documentación e Investigación de la Cultura de Izquierdas en Argentina (CeDInCI). Fue Herminia Brumana una mujer que "debió desplegar, además, estrategias específicas dadas por su género para poder avanzar en su profesión y también para poder acceder a otros espacios culturales, como la publicación de sus escritos en diversas revistas y periódicos". ${ }^{8}$

Dentro de los ideales más destacados del pensamiento de Brumana podemos mencionar su concepción de maternidad como fundamento de lo femenino. "Ella reivindica la maternidad como potencia nodal para la transformación social a través de la educación (y de la transmisión de valores contrarios a la guerra) de hijas e hijos (y alumnas y alumnos)". 9 Asimismo, deduce que en la escuela los saberes a ser considerados deben ser los que formen a los seres humanos en afectos, valores, sentimientos, emociones y con motivo de ir en busca de la igualdad.

"Para Brumana, es la maestra quien debe contactar a sus alumnos con un universo de saberes, pero también de valoraciones hacia los otros". ${ }^{10}$ Ella imagina escuelas con alumnos que luchen por una sociedad más justa e igualitaria. Como maestra, comparte a través de sus escritos con sus compañeros y adeptos las formas que ella supone convenientes para que esto suceda, a la vez que desde una postura comprometida con sus postulados aconseja al respecto, inspirada siempre en sus ideales.

\footnotetext{
${ }^{7}$ Lobato 216.

${ }^{8}$ Marina Becerra, Un prisma original: educación, género, amor y ciudadanía en Herminia Brumana, (Buenos Aires. Vol. 17. 2017). 2.

${ }^{9}$ Becerra 13.

${ }^{10}$ Paula Caldo, Herminia escribe para sus alumnos y alumnas. Notas sobre la experiencia de una maestra argentina, 1918. (XII CIHELA. Medellín. 2016) 7.
} 
"Herminia Brumana no solo interpretó las características de las mujeres que habitan en el imaginario escolar, sino que además fue una militante que se valió de la palabra escrita para alcanzar otros públicos lectores". ${ }^{11} \mathrm{Y}$ este es quizás uno de los motivos por los cuales se la pueda incluir bajo la expresión otros intelectuales que Flavia Fiorucci desentraña. Esta maestra, a la par de ejercer la docencia produjo conocimientos destinados a un amplio público lector y de temas candentes para la época (mujeres, educación). Lo hizo representando escenarios que mostraban el modo en que las condiciones materiales de la vida afectan a las prácticas, a las maneras de aprender y reclama mujeres que aborden, borden y desborden las escuelas "[...] sensibles, atentas, capaces de "mirar viendo" y de leer el mundo que rodea a sus alumnos y a ellas mismas". ${ }^{12}$

El nueve de enero del año 1954, a sus 57 años, Brumana fallece en la ciudad de Buenos Aires. Entre varias de las políticas de memoria que operaron sobre su labor, quedan guardadas las cartas que Herminia enviaba y recibía; algunas de las cuales analizaremos en breve. Las epístolas seleccionadas son aquellas que le fueron enviadas a la maestra en respuesta a las que ella remitía o las que se destinaban a su persona con motivo de abrir (sus lectores, otros intelectuales o colegas) al cambio de ideas. Esta maestra autora, al haber publicado sus escritos dejó marcas que en la actualidad pueden encontrarse no solo en reservorios públicos tales como bibliotecas, sino también, y en su caso en particular, en fondos documentales específicos. Algunas de estas notas serán utilizadas para dar lugar a un entrecruzamiento entre sus escritos, fundamentalmente su primer libro Palabritas, y lo que los estos generaron en quienes leían las publicaciones.

Podemos pensar entonces que el transcurso de la vida de Brumana tuvo lugar en uno de los momentos en los que gracias a las luchas sociales y políticas de las mujeres comenzaban a brindárseles algunas concesiones, las que obtenían luego de la toma de conciencia de la inferioridad de su condición como ser humano dentro de la sociedad. En los versos de Brumana asoman voces de honda protesta. Es un clamor vehemente por los niños que padecen frío y hambre y una casi airada defensa de la madre humillada por la torpeza de la gente. ${ }^{13}$

Brumana adhirió a muchos postulados de la militancia socialista, como el de intervenir en los debates sobre la situación de las trabajadoras y sobre los derechos laborales, del mismo modo que defender la consecución del derecho al sufragio o al divorcio. ${ }^{14}$ También entonces algunas propuestas de feminismo socialista reclamaron el reconocimiento de la identidad femenina como luchadora sindicalista y trabajadora ${ }^{15}$

\footnotetext{
${ }^{11}$ Caldo 3 .

${ }^{12}$ Caldo 11.

${ }^{13}$ Marta Elena Samatán, Herminia Brumana. La rebelde, Editorial Plus Ultra. Buenos Aires. 1974.

${ }^{14}$ Lobato 2007.

${ }^{15}$ Nash Mary. El mundo de las trabajadoras: identidades, cultura de género y espacios de actuación. En Paniagua, J; Piqueras, J; Sanz, V. (Editores) Cultura social y política en el mundo del trabajo. Biblioteca Historia Social, Valencia. Páginas 47-69. 1999.
} 
siendo a principios del siglo XX la "cuestión de la mujer" parte de la "cuestión social", dado que las transformaciones ocurridas a las mujeres eran explicadas socialmente (producto de transformaciones económicas, del advenimiento del capitalismo) y porque uno de los cambios más dramáticos pasaba por su incorporación al mercado de trabajo. ${ }^{16}$

Aquí encuadramos el pensamiento de Brumana; ella no diciéndose formar parte del movimiento feminista sí se pensaba luchadora por los derechos de los trabajadores. Leemos en una entrevista en La Literatura Argentina del mes de octubre de 1929 que decía: "Yo soy una modesta y simple trabajadora como ese hombre que allí levanta esa pared. Trabajo como maestra. Soy una obrera, nada más, y de ello estoy más orgullosa que si ostentara títulos nobiliarios o me confundieran con tanta gente cuyas vidas vacías e inútiles se pierden en los recodos de los escarceos amorosos o en el deporte del fácil elogio y del chisme ligero". ${ }^{17}$ Un aspecto conflictivo de la construcción de la ciudadanía en la Argentina puede tenerse en cuenta aquí si se observa a partir de un componente señalado como complementario de la ciudadanía política y social: el reconocimiento de derechos y deberes de los trabajadores, con sus diferencias de "género", junto a la aceptación de beneficios que implicarían la obtención de mayor bienestar entendidos como integrantes de la ciudadanía social. ${ }^{18}$

Sabido es que, hacia fines del siglo XIX y principios del XX, se suponía la existencia de una diferencia fundante de varones y mujeres; de caracteres anatómicos, fisiológicos, rasgos psíquicos, espirituales y morales. ${ }^{19}$ Las mujeres quedaban excluidas de actividades que no refirieran a gestar, parir y amamantar a un nuevo ser humano. El trabajo femenino entonces no era un factor nuevo introducido por la "civilización moderna" o el capitalismo, aunque sí novedosa su elevación a problema social..$^{20}$ Podemos afirmar entonces que en los años veinte dentro de las labores asalariadas probables y posibles se encuentra la docencia; la que reafirmaba la naturaleza de las mujeres de tipo maternal.

Pensamos de este modo a Brumana autora de cuentos infantiles y libros pedagógicos, guionista de obras teatrales, novelista, quien publicó otros escritos varios en la prensa siempre desde su condición de maestra en el grado y en la gestión escolar. ${ }^{21}$ Fue docente por título (maestra normal) y por oficio, pero formó también parte de esos otros intelectuales, productores de conocimiento con claras proyecciones

\footnotetext{
${ }^{16}$ Marcela Nari, "El feminismo frente a la cuestión de la mujer en las primeras décadas del siglo XX". En Juan Suriano (compilador) La cuestión social en Argentina, 1870-1943. La colmena. Buenos Aires. (2000): 277-300.

${ }^{17}$ Fletcher Lea, Una mujer llamada Herminia, Bs. As, Catálogos Editora. 1987.

${ }_{18}$ Mirtha Lobato, "El Estado en los años treinta y el avance desigual de los derechos y la ciudadanía", Estudios Sociales. (7), (1997): 41-58.

${ }^{19}$ Marcela Nari, "Feminismo y diferencia sexual. Análisis de la "Encuesta feminista argentina" de 1919", Boletín del Instituto de Historia Argentina y Americana. Tercera serie (12), 1995.

${ }^{20}$ Nari 113.

${ }^{21}$ Graciela Queirolo, "Herminia Catalina Brumana. La maternidad social a través del magisterio y la escritura", Mujeres en espacios bonaerenses, EDULP. La Plata, (2009): 95-109.
} 
sociales y políticas, autónomos, críticos pero marginales a los centros de producción hegemónicos, generalmente metropolitanos y masculinos. ${ }^{22}$ A continuación, analizaremos una de sus múltiples intervenciones en el ámbito de lo público para seguir reflexionando sobre su condición de militante experimentada que, valiéndose de la palabra escrita, alcanzó grandes públicos lectores logrando impactar en la sensibilidad de los mismos para provocarles acción, tal es el caso de su recorrido en la prensa educativa y propuesta editorial para docentes y alumnos, a quienes incitaba a construir formas igualitarias de la vida en sociedad.

\section{Palabritas: una obra que destaca el impacto del clima de la época en el pensamiento y oficio de la maestra}

Palabritas, primera experiencia en la escritura de Herminia dirige sus relatos a niños de entre 11 y 13 años y lo hace de un modo particular: mediante una invitación a pensar en concepciones sobre "desigualdad", "libertad" y "trabajo". La autora recurre a estos términos y los carga de significado, realiza comparaciones entre las realidades de los pequeños dándole especial atención a situar como base de los conflictos sociales las desigualdades materiales que marcan y a la vez tensionan la sociedad. Con fuertes argumentos, debuta Herminia Brumana como escritora de libros para niños, pero no repara en el modo en que sus alumnos aprenden (lo cognitivo), sino en los contextos sociales desiguales que los marcan con sus condiciones de posibilidad para estudiar, jugar, alimentarse.

Lejos de ser un libro cargado de historias encantadoras y narraciones placenteras, Palabritas viene a discutir sobre temáticas tan complejas como las referentes a las desigualdades económicas, a la pobreza. Nos proponemos entonces, desde aquí y en adelante, indagar sobre el modo en que la autora plantea estos problemas con su libro, las reflexiones que una vez publicado giran en torno al mismo. Primeramente, creemos relevante mencionar que dicho ejemplar fue editado en los Talleres Gráficos Argentinos L.J. Rosso (Buenos Aires), cuando corría el año 1918. En palabras de la autora: "Lo he hecho para mis alumnos, mil ejemplares me han costado 300 pesos que he juntado moneda a moneda, privándome de un vestido, de una cinta, de un adorno". ${ }^{23}$ J. Palabritas es un libro destinado a los niños escolarizados. La maestra ubica a estos receptores desde el instante en que presenta las historias narradas dentro de la obra; intuimos Herminia encuentra más sencillo el ejercicio de escribir un libro dirigido a los infantes que realizar la misma tarea para las escuelas.

Encontramos en el primer relato del compendio, que se llama "Un día de frío" la siguiente sentencia: "En la escuela, el frío ha entrado y reina. En primero y segundo grado hay chicos que lloran. Cuando la maestra dicta los deberes para el día

\footnotetext{
${ }^{22}$ Flavia Fiorucci, "Presentación. Dossier: los otros intelectuales: curas, maestros, intelectuales de pueblo, periodistas y autodidácticas", Prismas, Nº 17, 2013.

${ }^{23}$ Juan Francisco Jauregui, "Herminia C Brumana, maestra en Pigüé”, disertación radial, 1956, 12 en Fondo Bibliográfico Herminia Brumana - Centro de Documentación e Investigación de la Cultura de Izquierdas (FBHB/CEDINCI).
} 
siguiente, brillan alegremente los ojos. ¡Siquiera pueden irse, llegar a la casa, donde la madrecita espera con el té bien caliente!". ${ }^{24}$ A lo que sigue párrafos después: "Eso pensáis vosotros, que tenéis un hogar cómodo... Hay otros chicos que, en estos días de frío, cuando la maestra dicta los deberes, sienten ganas de llorar. Y esos niños terminan ese día de frío acostados en un catre, con poco abrigo [...]". ${ }^{25}$

Ubicar en el libro Palabritas el modo en que la autora plantea el problema de la desigualdad social, nos conduce a trabajar sobre los ejemplos que nos muestran a la maestra recitando escritos para alumnos que los ayuden a reflexionar sobre temáticas tan debatibles como la de la desigualdad material. Como decíamos antes, la docente en su primer libro no se pregunta por el modo en que sus alumnos aprenden, ella se muestra interesada por los contextos sociales desiguales que marcan diferencias entre los niños, contextos dispuestos por contrastes materiales que ella pretende conozcan los más pequeños y reflexionen al respecto. Brumana reitera la condición de niños pobres y ricos, de aquellos que llegan a sus hogares una vez finalizada la jornada escolar y cuentan con un plato de comida saludable y el resto de su día destinado al descanso y aquellos que no gozan de ese beneficio: chicos que trabajan ayudando a sus familias porque lo necesitan.

Aclarando que Palabritas es un libro para escolares y no para niños (cuentos con los que considera al docente figura indicada para saberlos utilizar, según el modo que éste suponga correcto y aquel que le permita hacerlos más provechosos para su particular grupo de alumnos), en "Un juego" Brumana describe: "Yo te voy a decir: juega a la chacrita. ¿Sabes cómo es? Bueno; para eso tienes que pedirle a tu padre un pedazo de patio, allá en el fondo, junto a la pared [...]". ${ }^{26} \mathrm{Y}$ en párrafos siguientes continúa: "Si tienes hermanitos, mejor; serán tus compañeros de trabajo, tus socios. Óyeme bien: tus socios, no tus peones. La chacrita será de todos. Ya notarás cómo te gusta, cómo te hará bien andar con la pala y la azada $[\ldots]$ ". ${ }^{27}$

El primer libro de Brumana, con estilo sucinto y a la vez inquietante, incluía al interior la orientación humana y justiciera que mostrará en toda su labor posterior. La obra se encontraba destinada a la lectura de los grados superiores, y quizás la peculiaridad que presenta en sus relatos es la que no le permite la aceptación para ser usada en las instituciones educativas (libro desaprobado por el concurso público que regía bajo la Ley de Educación Común $N^{\circ} 1420$ antes mencionada). De cualquier forma, su primer escrito fue publicado por la autora en el año 1918 y entregado a modo de obsequio a compañeros y conocidos del mundo letrado. Al ser esta su primera experiencia de escritura editorial, la autora en Palabritas compone una serie de relatos breves con el fin de recrear las diferencias sociales que separan y marcan a los niños y a las niñas. En tales relatos la invitación es pensar el sentido de la pobreza, de las mesas sin comida, de las diferencias sociales, de las situaciones de opresión

\footnotetext{
${ }^{24}$ Herminia Brumana, Palabritas, (Talleres Gráficos Argentinos. L.J. Rosso. Buenos Aires. 1918): 1-37

${ }^{25}$ Brumana 14.

${ }^{26}$ Brumana 15.

${ }^{27}$ Brumana 16.
} 
y falta de libertad. Es un libro que trabaja las nociones de igualdad y de libertad a partir de recrear historias donde tales valores están ausentes. Podemos imaginar que en el contenido está el motivo de la no aprobación del texto para ser difundido en las escuelas argentinas.

En el deseo de la escritora anida la intención de despertar conciencias, haciendo que se tomen en consideración y piensen las diferencias socioculturales, en especial por aquellos que las desconocen por no padecerlas. El trabajo, el hambre, la soledad, la opresión y sentimientos negativos (la envidia) son temas ejes en el texto. Vemos entonces el modo en el cual la no aprobación oficial del libro para ser distribuido en las escuelas se debió al contenido inadecuado del texto para los años de escolaridad indicados. Tal motivo no prohibió a la maestra difundirlo entre sus colegas y amigos; ella así expresó la lucha con la escritura como arma principal por el triunfo de sus nobles ideas, formando parte de aquel grupo de mujeres que en la primera mitad del siglo XX participaba en el debate, entre otros temas candentes, sobre la condición femenina en la sociedad moderna.

Por otra parte, analizar el contenido del libro teniendo presente el contexto social en el cual se circunscribe, y como material didáctico el impacto que genera nos invita a leer cartas que mostraban opiniones sobre los ejemplares (las que recibía Herminia Brumana de aquellas personas favorecidas con la donación de un ejemplar y que ella misma luego respondía). Dicha correspondencia nos ayuda a percibir las expresiones de quienes aludían acerca de las opiniones que Palabritas suscitaba en los lugares en los cuales había sido difundido, junto a las discusiones que afloraban con la aparición de la primera obra de la maestra.

Juana de Ibarbourou, reconocida intelectual con la cual Brumana compartía espacios del mundo letrado escribe: "Encantada con "Palabritas". Palabritas que son grandes palabras en el fondo, de tan pura y emocionada moral. La abraza su amiga. Juana". Al igual que el escrito de esta reconocida erudita leemos otro del cual no identificamos la firma y suponemos es de un colega (maestro) o amigo: "Palabritas. Este, como todo lo que usted escribe, yo no sé si porque me impresionan hondamente sus cosas o porque ha dado la casualidad de que siempre la he leído cuando más abatido se ha encontrado mi espíritu, que le aseguro que leyéndola se me forma un nudo en la garganta y es todo lo que puedo decirle [...]”.

"Acabo de leer sus "Palabritas", y a trueque de parecer osado le envío la presente con la que quiero testimoniar mi profunda adhesión a la causa que Ud. defiende: sembrando en el corazón de la niñez semillas de verdad y amor. Tengo fe en el triunfo que es para los obreros del ideal: sean los de músculos de acero o los de plumas de oro." Le escribe el doctor Ernesto Pedrazzini, en Saavedra el 23 de noviembre de 1918.

A su vez, el Profesor Normal C. U. Videla Rivero, con fecha 2 de noviembre de 1918 y en la ciudad de Olavarría expresa: "Oportunamente recibí Palabritas... ante todo, mis agradecimientos. Su librito me gusta por su fondo humano, hondo a veces 
por el significado y alto por la inspiración moral que lo guía. Me gusta por su estilo: sencillo y natural, sin los pueriles embelecos literarios de los que sintieron o pensaron poco..." En estos fragmentos de epístolas recibidas por Brumana reconocemos el acuerdo por parte de un sinnúmero de figuras cercanas a la autora con respecto al contenido de la obra. Todos felicitan a la maestra por su escrito, inicial de otros que la acompañarán a lo largo de su carrera como literata y que dan muestra de una educadora animosa de desnudar prácticas cotidianas que pasaron, y podemos afirmar aún siguen pasando, desapercibidas y se encuentran sujetas a una apariencia inocente que reproduce el dominio. Ya los primeros escritos de Herminia Brumana demostraban su compromiso social, posicionamiento político e interés en lo cultural.

La autora con su libro pretende abrir a la discusión entre los chicos sobre temas centrales para la época; Palabritas será desacreditado (seguramente por su tendencia liberal) por el entonces Consejo General de Escuelas de la provincia de Buenos Aires (con fecha 30 de mayo 1931), para la distribución en las escuelas públicas, pero se hará famoso por la admiración que sus colegas y amigos expresarán al recibirlo.

Bien sabido es que Palabritas es redactado por Brumana a comienzos del siglo XX; desde su filiación anarco-socialista como periodista, dramaturga y activista de los derechos de la mujer, la autora con su texto pretende revisar, sin tapujos, la realidad social americana en la que se destaca la necesidad de una educación que fomente el pensamiento crítico en los niños. En este texto analiza el territorio escolar y se anima a hacer lo mismo con el social, para interpretar ambos atendiendo a sus prácticas, y con el fin último de ayudar a los estudiantes a desnaturalizar algunas inadecuadas conductas.

Su relato muestra el lugar donde las realidades se hacen visibles, lugar de heterogeneidad, diversidad que pide ser atendida. Como maestra, se niega a disimular las contradicciones que aparecen en los diálogos cotidianos y propone a los educandos la lectura de fragmentos conformados por relatos, reflexiones, ensayos que acompañan a los chicos a comprender las realidades que los atraviesan, constituidas estas por un sinnúmero de diferencias, desigualdades que la autora considera se encuentran encubiertas y quiere lograr descubrirlas y manifestarlas para comprenderlas junto a sus alumnos. La maestra se inicia en el ejercicio de la docencia utilizando dicha práctica educativa para escribir, publicar, hacer conocer su modo de pensar.

Además, el clima de entreguerras puso en circulación saberes oficiales sobre la docencia, a la vez que habilitó bordes por donde se expresaron en forma genuina otras voces, entre ellas, las de las maestras. ${ }^{28}$ Siendo fuentes de consulta frecuente en la historia de la educación, las revistas pedagógicas proliferaron en el devenir de la primera mitad del siglo XX. Y una de ellas, de carácter mensual y procedencia

\footnotetext{
${ }^{28}$ Pellegrini Malpiedi Caldo, "Scribere est agere..., o un espacio impreso para dar a leer y a ver a las maestras y a las estudiantes de Magisterio, Rosario, 1925-1930”, en Fernández, S. (Coordinadora) Temas de historia social santafesina (principios del siglo XX), Ediciones del ISHIR. Rosario. 2015.
} 
santafesina: llamada Simiente, convoca diez años después de la primera edición de Palabritas a dejar algunos fragmentos de ese escrito en la publicación. Encontrando ejemplares de Simiente en la Biblioteca Pedagógica Eudoro Díaz (de la ciudad de Rosario), de 1928 a 1932, identificamos que la misma pertenecía al órgano de la Sociedad Unión del Magisterio.

Llamándose periódico vocero del maestro y defensor de la educación pública de la provincia de Santa Fe, en su número donde publica un fragmento de Palabritas Brumana, leemos el apartado titulado: Mientras reina la primavera. Allí la maestra aconsejará a los más pequeños aprovechar de dicha estación para hacer el bien, y no esperar la llegada del invierno para brindar como el árbol sombra, consuelo y apoyo para quienes lo precisen. “... donde las flores y los frutos (las buenas obras) lleguen abundantes y repartidas sin mirar a quiénes". ${ }^{29}$

Será entonces Palabritas un libro de uso en bibliotecas populares y/o personales, obra que marcará el inicio de la labor de una mujer dedicada al magisterio, pero confiada en que la educación favorecería a la multiplicación de acciones humanitarias, en busca de una sociedad más justa. Será también un escrito que Brumana elegirá para utilizar una década después en diversos espacios culturales $\mathrm{y}$, con el que difundirá sus ideas en (por ejemplo) la primera revista del gremio docente de la provincia argentina de Santa Fe: Simiente. Revista que además de aportar preceptos para la teoría y la práctica educativas, presentaba sugerencias, inquietudes, desafíos acerca del magisterio, junto a construcciones discusivas marcadas por las condiciones de posibilidad de la época y que contenían algunos indicios que permitían leer singularidades femeninas: problemas salariales, el mundo del trabajo, la formalización de la educación femenina.

El equipo de redactores de Simiente se encontraba conformado por personalidades que formaban parte de la comisión directiva de la Unión del Magisterio de Santa Fe. En dicha revista, y con un relato sobre la primavera, Brumana fomentará desde la educación el pensamiento crítico en los más pequeños. Y, al igual que ella, otras mujeres destinarán escritos a sus colegas del magisterio, por lo que interpretamos Simiente convocaba a las pedagogas a escribir. Allí, aparece la arriba mencionada poetisa uruguaya Juana de Ibarborou (1892-1979) y la ensayista y crítica literaria rosarina Ana María Benito (1900-1930).

Por otra parte, y volviendo a Palabritas, obra que en su apertura temática da a conocer una gran variedad de lecturas dirigidas a los estudiantes, observamos en uno de sus escritos el hincapié que realiza la autora cuando remarca bajo el concepto de trabajo igualitario la diferencia de género. El texto propone actividades a niños por un lado y niñas por otro; allí se advierte como destino indiscutido de la mujer la maternidad, como posibilidad de orden biológico, pero al mismo tiempo habilitante de intervenciones históricas y sociales. La condición femenina de parir

\footnotetext{
${ }^{29}$ Herminia Brumana. Obras Completas. (1958). Compilación y prólogo de José Rodríguez Tarditi. Edición Amigos de Herminia Brumana. Buenos Aires.
} 
sumada el gesto social de criar en calidad de madre, fueron denotando un núcleo de lucha e identificación femenina defendido y reconocido por las mujeres adheridas al anarquismo, por militantes socialistas, movimiento social el último en el que incluimos a la maestra aquí estudiada.

Brumana fue una mujer dedicada al magisterio, con vocación de educadora y elevado sentido de misión social, acreditada con espíritu renovador y progresista. Como sabemos, la Ley argentina de Educación Común 1420 estableció la legalidad de la educación obligatoria tanto para varones como para mujeres, oficializando la asistencia femenina. Al menos en Argentina, las mujeres encontraron en el ejercicio de la docencia una posibilidad de extender su rol doméstico a una institución pública a la vez que trabajar, escribir, percibir un salario. Brumana así lo hizo y, como vimos, a través de la escritura ${ }^{30}$ reflexionó sobre el problema de las mujeres en el ámbito de lo público dejando de suponer que la división sexual del trabajo separaba "mundo privado" femenino de "mundo público" masculino, aceptándose que algunas tareas eran más "femeninas" que otras y, por lo tanto, eran más legítimas para las mujeres. La labor de esta mujer, dedicada al magisterio y a la escritura, de lucha por la defensa de los derechos humanos, podemos localizarla aquí bajo la narración de libros de texto para los más pequeños. Palabritas será una obra relevante en la vida de Brumana. Ella usará muchos años después fragmentos de su escrito para reflejar en revistas de gremios del magisterio, como observamos, sus firmes ideales. Tal como la maestra lo mencionó en más de una oportunidad, escribir para ella era una herramienta para contribuir en la realización de un mundo mejor.

Por otra parte, y si bien Herminia Brumana venía participando desde su escritura en distintos proyectos editoriales anarquistas, la década del 30 la encuentra profundamente comprometida con las ideas y las organizaciones libertarias. "Podemos encontrar el nacimiento de esas ideas en Palabritas, un hermoso libro que a pesar de ello no se ajustaba a los cánones que regían por esa época". ${ }^{31}$ Tampoco se había solicitado para el mismo la aprobación de las autoridades escolares. El texto de Brumana no salió airoso de la evaluación, quedando así inhabilitada su aplicación en las aulas. De todos modos, esta obra lejos de ser diminuta venía a poner en Palabritas una serie de tensiones difícilmente resueltas en el interior del mundo de las prácticas educativas, motivo por el cual no obtuvo la tan deseada aprobación para su distribución en las escuelas públicas argentinas.

En las páginas del libro había motivos suficientes para que el Consejo Escolar de Distrito no aceptara su circulación en las escuelas. “¿Cómo no iba a escandalizarse dicho Consejo frente a esa maestra que desdeñaba al odioso fabricante de fusiles, esos malditos fusiles que, para muchos, son todavía el pedestal de la futura grandeza de la patria?" 32 Quizás fue la primera obra literaria de Herminia Brumana aquella causante de dar apertura a la búsqueda incesante y convencida, por parte de la maestra, de luchar con la escritura como arma principal por el triunfo de sus nobles ideas.

\footnotetext{
${ }^{30}$ Nari 277.

${ }^{31}$ Marta Samatán, Herminia Brumana. La rebelde. (Buenos Aires, Editorial Plus Ultra 1974) 13

${ }^{32}$ Samatán 14.
} 


\section{Algunas reflexiones finales}

Si retomamos las ideas mencionadas al inicio del escrito que refieren a los sucesos ocurridos en la Revolución Rusa y que se relacionan íntimamente con la emancipación de la mujer podemos pensar en el análisis que aquí efectuamos sobre la obra inicial de una maestra argentina que, en su desempeño como docente, se volvió emblemática; Herminia Brumana fue maestra, escritora y militante, ${ }^{33}$ cuya labor transcurrió en la primera mitad del siglo XX argentino. Su biografía ejemplifica el perfil intelectual de las educacionistas argentinas de antaño. Fue docente por título y por oficio, pero fue intelectual en virtud de que sus intervenciones públicas estaban legitimadas por el pensamiento crítico, reflexivo e independiente. ${ }^{34}$

Si teniendo en cuenta el mismo período, nos trasladamos a la realidad europea y apreciamos las conquistas que la Revolución Bolchevique del año 1917 hizo posibles, nos encontramos con la real obtención de igualdad económica de mujeres y hombres, suceso que impactó en el resto del mundo, al intentar llevar a la mujer a la participación plena en la vida social, económica, política y cultural.

A partir de entonces comenzaron a visualizarse beneficios para las mujeres; en Rusia el movimiento feminista era parte de la corriente democrático-burguesa más amplia que se oponía al zarismo y quería modernizar al pueblo ruso como sociedad capitalista industrial. Justamente la causa revolucionaria en Rusia echó raíz debido al despertar político de las mujeres trabajadoras de la ciudad y la aldea frente a su misión histórica. Por debajo de este contexto mundial encontramos motivos suficientes para animarnos a escribir sobre una maestra argentina que en el período desafió los postulados establecidos y, bajo su condición de trabajadora en el ámbito de la enseñanza, usó sus habilidades para introducir debates que en ese entonces no eran comunes de ingresar a las discusiones de interés social.

Herminia Brumana aparece aquí como ejemplo factible para recordar mediante el presente escrito a las mujeres de aquellos años y reivindicar a las primeras figuras femeninas que trabajaron en oficios no solo para aparecer en el ámbito de lo público sino también para luchar por sus derechos, la obtención de libertades, el pleno y verdadero acceso a los dominios culturales y económicos y, por sobre todas las cosas, olvidando la división de los sexos para aunarse bajo una condición que era la de trabajador y bregar por la lucha de clases de todos los explotados contra sus explotadores.

Utilizar aquí el pensamiento de Herminia resulta un mirador adecuado para estudiar cómo algunas maestras, junto a otros actores del ámbito de la subalternidad,

\footnotetext{
${ }^{33}$ Ver: Lucía Lionetti, "Las no ciudadanas en la plaza pública. Voces y acciones de educadoras, escritoras y militantes”, en Educación, género y ciudadanía. Las mujeres argentinas: 1700-1943, comp. Pilar Pérez Cantó y Susana Bandieri, (Buenos Aires: Miño y Dávila, 2007): 225-272.

${ }^{34}$ Federico Neiburg y Mariano Plotkin, comp. Intelectuales y expertos. La construcción del conocimiento social en argentina, (Buenos Aires: Paidós, 2004): 15.
} 
muchas veces lograron hacerse visibles gracias a sus capacidades y el capital social del cual eran portadoras. Brumana alude a una maestra situada en un contexto que incluye tanto su vida cotidiana y afectiva, como la de los alumnos y la del universo escolar a pleno. La autora decide enlazar ambos registros para pensar formas diferentes de interpretar y proyectar el oficio docente.

Durante mucho tiempo, la historia de la educación indicó sus agentes bajo un universal genérico inscripto con los trazos de la división de género propia de la sociedad patriarcal y heterosexual. Por lo cual, una línea historiográfica de reciente data brega por visibilizar la agencia específica de las maestras, y es en esta dirección que recuperamos la figura de Herminia Brumana para distinguir el modo en que, dentro de las voces del magisterio, pronunció agudas críticas configurándose como maestra intelectual comprometida con su oficio. Bajo esta línea, un sinnúmero de escritores remarca la importancia de la construcción simbólica de la mujer como madre de la Nación en la legitimación de la República. La influencia del positivismo compteano es evidente en este aspecto. En la escala de valores el primer lugar era para la humanidad, luego la patria y después la familia. La mujer representaba idealmente los tres estadios como madre, lo cual le asignaba un rol primordial en el ámbito privado del hogar y una inclusión real casi nula en el espacio público. Así, la familia era una de las instituciones de la sociedad civil y tanto dentro como fuera de ella las mujeres tenían una participación importante y clave en los procesos nacionales. Ellas estaban involucradas en la reproducción ideológica como transmisoras de cultura, participaban en los conflictos sociales.

Desde 1890, y con motivo de edificación de una sociedad moderna, la cuestión familiar aparece en la base de dicha construcción, operando la mujer en los planos político y laboral. Herminia Brumana fue madre, esposa, escritora y militante. También docente por título y por oficio, pero también fue intelectual en virtud de que sus intervenciones públicas estaban legitimadas por el pensamiento crítico, como así también por el mercado editorial metropolitano. Brumana dictaba clases y cumplía con las prescripciones escolares. Al mismo tiempo, escribía y publicaba sus textos en formato de libro y de artículos periodísticos.

Brumana adhirió a muchos postulados de la militancia socialista, tanto femenina como masculina, “[...] como el de intervenir en los debates sobre la situación de las trabajadoras y sobre los derechos laborales, del mismo modo que defender la consecución del derecho al sufragio o al divorcio". ${ }^{35}$ Asimismo, y por aquel entonces, "[...] algunas propuestas de feminismo socialista reclamaron el reconocimiento de la identidad femenina como luchadora sindicalista y trabajadora". ${ }^{36}$

Por otra parte, muchos de los partidos políticos del período llamaban a participar de la Jornada Internacional de la Mujer Trabajadora que organizaban,

\footnotetext{
${ }^{35}$ Lobato 215.

${ }^{36}$ Mary Nash, "El mundo de las trabajadoras”, En Paniagua, J; Piqueras, J; Sanz, V. (Editores) Cultura social y política en el mundo del trabajo. (Valencia. Biblioteca Historia Social. 1999): 66.
} 
acorde con la conmemoración que también se realizaba en Rusia desde que en 1917 las mujeres se declararon en huelga demandando "pan y paz". Aunque hay debates sobre los orígenes de la conmemoración, se sabe también que, con anterioridad, Clara Zetkin propuso su establecimiento en la Conferencia de Mujeres Socialistas, realizada en Copenhague en 1910. "Esta era una instancia de representación a escala internacional de la mujer obrera que demandaba por sus derechos y el de todas las mujeres como el derecho al voto, a ocupar cargos públicos, al trabajo, a la formación profesional y a la igualdad laboral" ${ }^{37}$

Optamos, en gran parte del presente escrito, por pensar en la Revolución Rusa porque la misma Brumana leía obras de referentes de la revuelta, como el caso de Alejandra Kollontai. Distinguimos esto, por ejemplo, en declaraciones de una entrevista realizada a la maestra en La Literatura Argentina en 1929 (Año II, No14, p. 46): “[...] He leído La bolchevique enamorada de Alejandra Kollontay, gran escritora rusa. "En ella se describe una mujer fuerte y moderna, pero no moderna porque fume, maneja auto o beba copetines sino porque siente inquietud de mejorar la condición de los humildes. Hermosa novela, desearía que la leyeran nuestras mujeres". ${ }^{38}$

Sabido es que a principios del siglo XX la cuestión de la mujer formó parte de la cuestión social, por lo menos, en dos sentidos: porque las transformaciones ocurridas a las mujeres eran explicadas socialmente: producto de transformaciones económicas, del advenimiento del capitalismo, de la industrialización. “[...] Por otro, porque uno de los cambios más dramáticos pasaba por su incorporación al mercado de trabajo, supuestamente reciente". ${ }^{39}$ Efectivamente a principios de siglo XX la lucha por reformas legales (igualitarias unas, proteccionistas otras), la interpelación al Estado fue encabezada por mujeres socialistas. En su mayoría, las feministas participaban en partidos y movimientos políticos-ideológicos. Aunque Brumana no se definía como maestra socialista, sí se muestra con pensamiento y sensibilidad social e invita a sus colegas docentes a humanizar la labor educativa para transformar la realidad de los estudiantes más pequeños.

Al igual que el feminismo, la "cuestión de la mujer" también comprendía una complejidad práctica y de sentidos. Para algunos, se vinculaba estrechamente al feminismo y a la liberación de las mujeres. Sin embargo, más comúnmente, se refería al "problema" de las mujeres en lo "público". Se suponía que la división sexual del trabajo separaba un "mundo privado" femenino y un "mundo público" masculino. Se aceptaba que algunas tareas eran más "femeninas" que otras y, por lo tanto, eran más legítimas para las mujeres: “[...] prácticamente, nadie discutía el empleo de mujeres en la docencia de los primeros grados; pero las feministas extendieron estas propuestas hacia ámbitos más conflictivos: la escritura profesional, el periodismo, las profesiones liberales". ${ }^{40}$ Un ejemplo de ello fue Herminia Brumana.

\footnotetext{
${ }^{37}$ Lobato Mirta. Historia de las trabajadoras en la Argentina (1869-1960). (Bs. As. Edhasa, 2007). 256

${ }^{38}$ Fletcher Lea. Una mujer llamada Herminia. (Buenos Aires, Catálogos Editora) 1987; 35.

${ }^{39}$ Nari Marcela. El feminismo frente a la cuestión de la mujer en las primeras décadas del siglo XX. En Juan Suriano (compilador) La cuestión social en Argentina, 1870-1943. (Bs. As. La colmena. 2000): 277.

${ }^{40}$ Nari 292.
} 
Vimos en Palabritas un sencillo libro que relata profundas verdades, pudiendo considerarlo el inicio de la labor de una maestra que confía en la educación para comenzar su acción humanitaria; “[...] docente que piensa en los niños sin protección, en los enfermos sin auxilio, para comprender las realidades sociales y a partir de las mismas reforzar su afán por la justicia, la verdad y el bien". 41 "Palabritas será fruto de una temprana vocación que se cumpliría en la vida de Brumana ampliamente: enseñar, alentar, orientar, presentar la vida sin asperezas y sin cobardías". ${ }^{42}$

Esta obra muestra un criterio didáctico que fija un modo particular de desarrollar en adelante su profesión de escritora; al mismo tiempo que enseña, Herminia Brumana pretende impactar en el corazón de los pequeños lectores a quienes se dirige encauzada siempre con su propósito ulterior, que es el de orientarlos hacia el camino del bien.

Herminia Brumana, en una entrevista en La Literatura Argentina del mes de octubre de 1929 decía: "Yo soy una modesta y simple trabajadora como ese hombre que allí levanta esa pared. Trabajo como maestra. Soy una obrera y de ello estoy más orgullosa que si ostentara títulos nobiliarios [...]". ${ }^{43}$ Quien se convertiría en prolífica escritora de textos de orden público, en sus inicios tan solo pretende (y aquí lo analizamos con Palabritas) repensar los espacios escolares entendiendo el dolor ajeno (particularmente vivenciado por sus alumnos que sufren las consecuencias de las desigualdades presentes en la sociedad del período) emitiendo juicios sobre las propias prácticas educativas.

Sacar a las mujeres de las sombras implica discutir con ciertos presupuestos conceptuales de las culturas políticas liberales del siglo XIX, como así también pensar en la ausencia en la esfera ciudadana de la mujer a lo largo de la historia identificando su rol, el que hasta mediados del 1900 estuvo marcado por el vínculo inicial con la naturaleza; siendo los varones capaces en lo racional, las mujeres no se encontraron habilitadas a introducirse en la esfera de lo político. Así, nuestras búsquedas y las fuentes a las que apelamos nos han posibilitado observar cómo en diversos espacios, con estrategias diferenciales, mujeres como la maestra Herminia Brumana, lograron resignificar sus prácticas en relación con el poder, situarse en un lugar diferente, aun cuando el sistema de dominación se encontrara en manos de los hombres.

En Argentina, sabido es que la feminización del magisterio fue construida dentro de límites de un régimen a cargo del género masculino, que reafirmaba una contradicción efectiva donde se pretendía hacer a las mujeres responsables de crear al ciudadano mientras que estas eran privadas del derecho a una ciudadanía plena; hecho que fue promovido por el propio Estado argentino desde las últimas

\footnotetext{
${ }^{41}$ Juan Francisco Jáuregui, "Disertación radial”. Herminia C. Brumana. Maestra en Pigüé. (CeDInCI. Buenos Aires. 1956): 10.

${ }^{42}$ José Rodríguez Tarditi, Herminia C. Brumana: escritora y maestra, (Artes Gráficas Modernas. Buenos Aires: 1956): 5.

${ }^{43}$ Fletcher Lea, Una mujer llamada Herminia, (Catálogos Editora, Buenos Aires, 1987): 43.
} 
décadas del siglo XIX por razones ideológicas y económicas. Pudimos determinar mediante el presente escrito cómo a este sistema educativo nacional se le escaparon excepciones a la regla y una de ellas fue Brumana; una maestra restringida en sus derechos civiles y políticos, pero habilitada a educar al ciudadano a la par de gestar reflexiones, juicios, premisas, con lápiz y papel en mano (todos ellos creadores de categóricas opiniones sobre la enseñanza en las escuelas, la condición de la mujer, diversas problemáticas sociales).

Las intenciones del presente escrito pretenden provocar en el lector una reflexión que considere el modo en que las discusiones que se advertían a nivel mundial llegaban a nuestro país y, en el caso estudiado, hicieron posible indagar acerca de la labor de una mujer dedicada al magisterio y a la escritura que, haciendo uso del mismo para manifestar sus expresiones, por aquel entonces silenciadas, discutió sobre temas tan relevantes para el período como la educación, la niñez, la mujer, las injusticias sociales.

Brumana luchó por la defensa de los derechos humanos desde sus salones de clases, escribiendo libros de texto para la enseñanza de los más pequeños; como lo dijo en más de una oportunidad, escribir para ella era una herramienta para contribuir en la realización de un mundo más humano, más justo.

\section{Bibliografía}

\section{Fuentes primarias}

\section{Fuentes de archivo}

Cartas del epistolario de Herminia Brumana. Centro de Documentación e Investigación de la Cultura de Izquierdas en la Argentina (CeDInCI). Buenos Aires.

Herminia Brumana. Obras Completas. (1958). Compilación y prólogo de José Rodríguez Tarditi. Edición Amigos de Herminia Brumana. Buenos Aires.

Palabritas. 1918. Talleres Gráficos Argentinos. L.J. Rosso. Buenos Aires.

Revista Simiente. Perteneciente al Órgano de la Sociedad Unión del Magisterio. Santa Fe. (1928-1932). Biblioteca Pedagógica Eudoro Díaz. Ministerio de Educación. Gobierno de Santa Fe. Santa Fe 2917.

\section{Manuscritos de archivo}

Disertación radial por Juan Francisco Jáuregui. 1956. “Herminia C. Brumana. Maestra en Pigüé. Centro de Documentación e Investigación de la Cultura de Izquierdas en la Argentina (CeDInCI). Buenos Aires. 


\section{Fuentes secundarias}

\section{Libros}

Alliaud, Andrea. Los maestros y su historia. Los orígenes del magisterio argentino/ 1. Biblioteca política argentina. Estudios sobre educación. Centro Editor de América Latina. Buenos Aires. 1993.

Barrancos, Dora. Anarquismo, educación y costumbres en la Argentina de principios de siglo. Buenos Aires, Contrapunto. 1991.

Barrancos, Dora. (Comp.) Historia y género. Buenos Aires. CEAL. 1993.

Barrancos, Dora. Mujeres en la sociedad argentina. Una historia de cinco siglos. Buenos Aires. Sudamericana. 2007.

Chartier, Roger. Escribir las prácticas. Foucault, de Certeau, Marin. Manantial, Buenos Aires, 2001.

Farge, Arlette. Escribir. Agenda cultural. Alma Mater. Universidad de Antioquía. ISSN 0124-0854. No 193 . Noviembre. Fragmento tomado de la obra La atracción del archivo. Valencia. 2012.

Fletcher, Lea. Una mujer llamada Herminia. Bs. As, Catálogos Editora. 1987.

Galeana, Patricia. Colección Las Maestras de México. Maestras urbanas y rurales. Siglos XIX y XX. Instituto Nacional de Estudios Históricos de las Revoluciones de México. Secretaría de Cultura. Volumen 3. Estudios constitucionales. 2017.

Giordano Verónica. Ciudadanas Incapaces La construcción de los derechos civiles de las mujeres en Argentina, Brasil y Uruguay en el siglo XX. Teseo. Buenos Aires. 2012.

Lionetti, Lucía. La misión politica de la escuela pública. Formar a los ciudadanos de la república 1870-1916, Miño y Dávila. Buenos Aires. 2007.

Lobato, Mirta. Historia de las trabajadoras en la Argentina (1869-1960). Edhasa. Buenos Aires. 2007.

Morgade, Graciela. Mujeres en la educación. Género y docencia en la Argentina 1870-1930, Miño y Dávila. Buenos Aires. 1997.

Mouffe, Chantal. El retorno de lo político. Comunidad, ciudadanía, pluralismo, democracia radical. Paidós. Estado y Sociedad. Buenos Aires. 1999. 
Neiburg Federico y Mariano Plotkin, (Comp.) Intelectuales y expertos. La construcción del conocimiento social en argentina. Paidós. Buenos Aires. 2004.

Paniza, Delio. Semblanza de Herminia Brumana. Buenos Aires: Montiel. 1954.

Peréz Cantó, Pilar y Bandieri, Susana. (Comp.) Educación, género y ciudadanía. Las mujeres argentinas: 1700-1943. Miño y Dávila. Madrid. 2005.

Perrot, Michelle. Mi historia de las mujeres. FCE. Buenos Aires. 2008.

Plotkin, Mariano y Eduardo Zimmermann (Comp.) Los saberes del Estado, Edhasa. Buenos Aires. 2012.

Plotkin, Mariano y Eduardo Zimmermann (Comp.) Las prácticas del Estado. Sociedad y élites estatales en la Argentina del siglo XX. Edhasa. Buenos Aires. 2012.

Puiggrós, Adriana. Qué pasó en la educación argentina. Breve historia de la conquista hasta el menemismo. Kapelusz Editora. Buenos Aires. 1996.

Puiggrós, Adriana; Marengo, Roberto. Pedagogías: reflexiones y debates. Universidad Nacional de Quilmes Editorial. Bernal. Buenos Aires. 2013.

Rodríguez Tarditi, José. Herminia Catalina Brumana: escritora y maestra. Buenos Aires: Artes Gráficas Modernas. 1956.

Samatán, Marta Elena. Herminia Brumana. La rebelde. Editorial Plus Ultra. Buenos Aires. 1974.

Sardi, Valeria. El desconcierto de la interpretación. Historia de la lectura en la escuela primaria argentina entre 1900 y 1940. Colección ciencia y tecnología. Santa Fe: Universidad Nacional del Litoral. Santa Fe. 2010.

Vassallo, Jaqueline; De Paz Trueba, Yolanda; Caldo, Paula (Coordinadoras) Género y documentación. Relecturas sobre fuentes y archivos. Colección el mundo de ayer. Brujas. Córdoba. 2016.

Yannoulas, Silvia. Educar. ¿Una profesión de mujeres? La feminización del normalismo y la docencia. 1870-1930. Colección triángulos pedagógicos. Editorial Kapelusz. Buenos Aires. 1996.

\section{Capítulos de libros}

Andújar, Andrea. Capítulo V. "El Género de la Historia: aportes y desafíos para el estudio del pasado". En Cristina Viano (coord.). Miradas sobre la historia. Fragmentos de un recorrido. Prohistoria. Rosario. 2012. 
Barrancos, Dora. "Moral sexual, sexualidad y mujeres trabajadoras en el período de entreguerras", en Devoto, Fernando; Madero, Marta (comp.), Historia de la vida privada en la Argentina. Tomo 3. Buenos Aires, Taurus. 1999.

Caldo, Paula. "Tizas y Apuntes, costumbres en común. Maestras, libros y prácticas, Argentina década de 1930", en Fiorucci, F. y Rodríguez, L. (Comp.) Intelectuales de la educación y del Estado: maestros, médicos y arquitectos. Universidad Nacional de Quilmes. Buenos Aires. 2016.

Cucuzza, Héctor Rubén; Pineau, Pablo. Para una historia de la enseñanza de la lectura y escritura en Argentina. Del catecismo colonial a La Razón de Mi Vida. Capítulo X. Colotta, Pablo. Escritura y poder en la Escuela Nueva Argentina. Capítulo XI. Pineau, Pablo. ¿Para qué enseñar a leer? Cultura política y prácticas escolares de lectura en el período de entreguerras. Miño y Dávila editores. Buenos Aires, Argentina, 2002.

Depaepe, M; Simon, F. "Qui ascendit cum labore, descendit cum honore. Sobre el trabajo con las fuentes: consideraciones desde el taller sobre historia de la educación”, en Lanzarín Miranda, F; Galván Lafarga, L; Simon, F. Poder, Fe y Pedagogía. Historias de maestras mexicanas y belgas. U.A.M. México. 2014.

Lionetti, Lucía. "Las no ciudadanas en la plaza pública. Voces y acciones de educadoras, escritoras y militantes”. Pilar Pérez Cantó y Susana Bandieri (Comp.) Educación, género y ciudadanía. Las mujeres argentinas: 1700-1943. Miño y Dávila, Buenos Aires: Páginas 225-272. 2007.

Nari, Marcela María Alejandra. "El feminismo frente a la cuestión de la mujer en las primeras décadas del siglo XX". En Juan Suriano (comp.) La cuestión social en Argentina, 1870-1943. La colmena. Buenos Aires. Pp.277-300. 2000.

Nash, Mary. "El mundo de las trabajadoras: identidades, cultura de género y espacios de actuación”. En Paniagua, J; Piqueras, J; Sanz, V. (Editores) Cultura social y política en el mundo del trabajo. Biblioteca Historia Social, Valencia. Páginas 47-69. 1999.

Queirolo, Graciela. "Herminia Catalina Brumana. La maternidad social a través del magisterio y la escritura", en Valobra, Adriana. (Editora), Mujeres en espacios bonaerenses, EDULP. La Plata, pp. 95-109. 2009.

Viñao Frago, Antonio. "La escuela y la escolaridad como objetos históricos. Facetas y problemas de la historia de la educación". História da Educação, 25, 9-54. En Mainer, Juan (2008) (coord.) Pensar críticamente la educación escolar. Perspectivas y controversias historiográficas. Prensas universitarias de Zaragoza. Zaragoza.

\section{Artículos de revistas}

Barrancos, Dora. “Mujeres en la Argentina: un balance frente al bicentenario”. Revista de Trabajo. Año 6. Número 8. Enero/julio 2010. 
Becerra, Marina. "Un prisma original: educación, género, amor y ciudadanía en Herminia Brumana”. Anuario Sociedad Argentina de Historia de la Educación. Buenos Aires. Vol. 17: 80-103. 2017.

Biernat, Carolina y Queirolo, Graciela. "Mujeres, profesionalización y procesos de profesionalización en la Argentina y Brasil”. Anuario del Instituto de Historia Argentina, 18. (1): 9-27. 2018.

Billorou, María José. "Mujeres que trabajan. Las maestras pampeanas en la primera mitad del siglo XX”, Anuario de la Facultad de Ciencias Humanas, No XII. 2015.

Caldo, Paula. "No parecían mujeres, pero lo eran. La educación femenina de las maestras, Argentina 1920-1930”. En Historia y Sociedad N 26: 237-265. Medellín, Colombia. 2014.

Caldo, Paula. "Revistas, consumo, alimentación y saberes femeninos. La propuesta de Damas y Damitas. Argentina, 1939-1944". En Revista de Historia y Ciencias Sociales. (94): 210-239. 2016.

Fiorucci, Flavia "Presentación. Dossier: los otros intelectuales: curas, maestros, intelectuales de pueblo, periodistas y autodidácticas”. Prismas, revista de historia intelectual. $\mathrm{N}^{\mathrm{o}}$ 17. 112-129. 2013.

Fiorucci, Flavia. "País afeminado, proletariado feminista, mujeres inmorales e incapaces: la feminización del magisterio en disputa (1900-1920)". En Anuario de Historia de la Educación. 120-137. 2016.

Flecha García, Consuelo. "Fuentes para la historia de la educación de las mujeres". Revista de Enseñanza Universitaria. Sevilla. España. No19. 51-62. 2002.

Lobato, Mirtha. "El Estado en los años treinta y el avance desigual de los derechos y la ciudadanía”. Estudios Sociales. Año VII, número 12: 41-58. 1997.

López, Oresta. "Las maestras en la historia de la educación en México: contribuciones para hacerlas visibles”. Revista Electrónica Sinéctica. Núm. 28: 37-55. 2006.

Nari, Marcela. "Feminismo y diferencia sexual. Análisis de la "Encuesta feminista argentina" de 1919". Instituto de Historia Argentina y Americana. Número 12: 213-241. 1995.

\section{Tesis, documentos, ponencia y otros}

Caldo, Paula. "Herminia escribe para sus alumnos y alumnas. Notas sobre la experiencia de una maestra argentina, 1918”. XII CIHELA. Congreso Iberoamericano de Historia de la Educación Latinoamericana. Medellín. 2016. 
Caldo, Paula; Fernández, Sandra. "Biografía, historia y mujeres: la revisión de un vínculo complejo a partir del caso de Olga Cossettini, 1898-1987”. Avances del Cesor. Año VII, N7/2010. ISSN: 1415-3899. Nodo Centro de Estudios Sociales Regionales del ISHIR (Investigaciones Sociohistóricas Regionales) dependiente del Consejo de Investigaciones Científicas y Técnicas y de la Universidad Nacional de Rosario. Rosario. 2010.

Caldo, Paula; Pellegrini, Micaela; Mosso, Agustina. "Experiencias singulares de enseñanza de la historia en las escuelas primarias santafesinas, un estudio de caso (primera mitad del siglo XX)". En Actas de las XV Jornadas Nacionales y IV Internacionales de Enseñanza de la Historia. UNL, Santa Fe. 2014.

Caldo, P; Pellegrini Malpiedi, M. "Scribere est agere..., o un espacio impreso para dar a leer y a ver a las maestras y a las estudiantes de Magisterio, Rosario, 19251930”, en Fernández, S. (Coord.) Temas de historia social santafesina (principios del siglo XX), Ediciones del ISHIR. Rosario. 2015.

Valobra, Adriana. La ciudadanía politica femenina en la Argentina de la primera mitad de siglo XX: Aportes para una aproximación conceptual y recursos didácticos. Clío \& Asociados (14), 86-112. En Memoria Académica. 